\title{
Effect of cellobiose supplementation and dietary soluble fibre content on in vitro caecal fermentation of carbohydrate-rich substrates in rabbits
}

\author{
César Ocasio-Vega, Rodrigo Abad-Guamán, Rebeca Delgado, Rosa \\ Carabaño, María Dolores Carro and Javier García
}

\begin{abstract}
The in vitro caecal fermentation of five substrates low in starch and protein content $[\mathrm{D}-(+)$-glucose (GLU), D-cellobiose (CEL), sugar beet pectin (PEC), sugar beet pulp (SBP) and wheat straw (WS)] was investigated using soft faeces from rabbits receiving different levels of cellobiose and soluble fibre as inoculum. A total of 24 rabbits were supplemented 3 levels of cellobiose in the drinking water $(0.0,7.5$, $15.0 \mathrm{~g} / \mathrm{l})$ and fed two experimental diets containing either low soluble fibre (LSF) or high soluble fibre (HSF) levels $(84.0$ and $130 \mathrm{~g} / \mathrm{kg}$ dry matter). All substrates were subjected to a two-step pepsin/pancreatin in vitro pre-digestion, and the whole residue was used as substrate for the in vitro incubations. Gas production was measured until $144 \mathrm{~h}$, and volatile fatty acid (VFA) production was determined at $24 \mathrm{~h}$ incubation. Experimental treatments did not affect SBP fermentation and had only a subtle influence on fermentation of WS and GLU. In contrast, cellobiose supplementation $x$ donors' diet interactions were detected for most gas production parameters for CEL. Both the fractional gas production $(k)$ and maximal gas production rates were linearly increased $(p \leq 0.042)$ and the initial delay in the onset of gas production (Lag) linearly decreased $(p<0.001)$ by cellobiose supplementation with the HSF inoculum, with no differences between the 7.5 and 15.0 doses. In contrast, with the LSF inoculum cellobiose supplementation only affected $k$ values, which were quadratically increased $(p=0.043)$ and had maximal values for the 7.5 dose. A quadratic effect $(p \leq 0.018)$ of cellobiose supplementation was observed for total VFA production at $24 \mathrm{~h}$ when CEL and PEC were fermented, obtaining the maximal VFA production for the 7.5 dose of cellobiose. Total VFA production for CEL was greater with LSF than with HSF inoculum $(20.7$ vs. $12.9 \mathrm{mmol} / \mathrm{l}$; $p=0.014)$, but the opposite was found for WS $(3.97 \mathrm{vs} .6 .21 \mathrm{mmol} / \mathrm{l}$; $p=0.005)$. The use of LSF inoculum for CEL fermentation sharply reduced acetate $(p=0.001)$ and increased butyrate proportions $(p \leq 0.001)$ compared with the HSF inoculum. A positive relationship between total VFA caecal concentrations in rabbits receiving the same experimental treatments and in vitro values was only observed when WS was used as substrate $(r=0.90 ; p=0.015 ; n=6)$. The results suggest that experimental factors influenced the fermentative activity of caecal digesta, but the observed response differed with the incubated substrate, being the CEL the most affected.
\end{abstract}




\section{Introduction}

Rabbits require a minimal amount of dietary insoluble fibre to maintain a high rate of passage to prevent the accumulation of digesta in the caecum that reduces feed intake and impairs growth (Gidenne et al. 2010). The dietary soluble fibre has also demonstrated some benefits like those on rabbit intestinal health in rabbits affected by epizootic enteropathy (Martínez-Vallespín et al. 2011; Trocino et al. 2013). They might be related to the amount of fermentable fibre either in the small intestine or in the caecum (AbadGuamán et al. 2015). The hydrolysis of the fibrous fractions liberates low molecular weight sugars in the small intestine (Pedersen et al. 2015), which might play a relevant role on the intestinal health of the animals. In fact, in some studies the dietary supplementation with cello-oligosaccharides (usually containing a high proportion of cellobiose) has demonstrated a positive effect on intestinal microbiota, mucosal architecture and nutrient transport in the small intestine of piglets after weaning (Jiao et al. 2014) and improved intestinal microflora, morphology and barrier integrity in broilers (Song et al. 2013). Studies in rabbits are scarce, but in a recent work cellobiose supplementation exerted a positive effect on rabbit health when animals were fed a diet low in soluble fibre, which was attributed to the greater butyrate concentrations observed in the small intestine of cellulose-supplemented rabbits (Ocasio-Vega et al. 2018b). Butyrate protects against intestinal mucosal injury and has demonstrated to prevent colonisation of enteric pathogens in the gastrointestinal tract through the upregulation of expression of the epithelial antimicrobial peptide in rabbits (Raqib et al. 2006; Cushing et al. 2015). Conversely, an increase in mortality was observed in the same study (Ocasio-Vega et al. 2018b) when cellobiose was supplemented to rabbits fed a diet rich in soluble fibre.

The fibrous fraction that enters the lower digestive tract can be fermented by the intestinal microbiota, producing volatile fatty acids (VFA) among other final products. Determining VFA production in vivo is difficult and measuring VFA concentrations in the digesta at a fixed time point is not a good estimator of VFA production and/or absorption because individual VFA have different absorption rates (Von Engelhardt et al. 1989). In contrast, total VFA production can be easily measured in in vitro studies. The use of in vitro methodologies could also be useful to confirm the effects of dietary treatments observed in vivo on fermentation patterns and to investigate the fermentative activity of digesta from rabbits fed different diets. The aim of this work was to assess the caecal fermentation of different substrates using inocula from rabbits fed diets differing in soluble fibre content and supplemented with different levels of cellobiose. In addition, potential relationships between VFA concentrations and profile measured in vitro and those determined in the caecum of rabbits receiving the same experimental treatments were investigated. In vivo results have been reported by Ocasio-Vega et al. (2018b).

\section{Materials and methods}

All procedures involving animals were carried out in accordance with the Spanish guidelines for experimental animal protection (Royal Decree 53/2013 of February 1st on the protection of animals used for experimentation or other scientific purposes [Boletín Oficial del Estado 2013]) after being approved by the General Direction of Livestock and Agriculture of the Community of Madrid (Approval number 10/196334.9/15). 


\subsection{Dietary treatments and donor animals}

Six experimental treatments in a $3 \times 2$ factorial arrangement, with 3 cellobiose concentrations in drinking water and 2 dietary levels of soluble fibre, were used. The 3 concentrations of cellobiose were $0.0,7.5$ and $15.0 \mathrm{~g} / \mathrm{l}$, and were obtained by diluting Dcellobiose (CEL) (NPC Cello-Oligo, Nippon Paper Industries Co., Tokyo, Japan) in the drinking water. According to the manufacturer, CEL contained $96.6 \%$ cellobiose $\beta 1-4$, $1.9 \%$ cello-oligosaccharide, $1.5 \%$ glucose and no nitrogen content. Concentrations of cellobiose were selected to obtain a wider range than those used in previous studies conducted with poultry and pigs $(0.15-0.45 \%$ in the diet; Song et al. 2013; Jiao et al. 2014).

Two experimental antibiotic-free diets were formulated to differ in the concentration of both soluble fibre ( 84.0 and $130 \mathrm{~g} / \mathrm{kg}$ dry matter [DM] for the low soluble fibre (LSF) and high soluble fibre (HSF) diets, respectively) and starch (226 and $182 \mathrm{~g} / \mathrm{kg}$ DM, respectively). The experimental design resulted in six dietary treatments that were named LSF0, LSF7.5, LSF15, HSF0, HSF7.5 and HSF15. Ingredients and chemical composition of diets are indicated in Table 1.

A total of 24 hybrid rabbits (New Zealand White $\times$ Californian) were weaned at $34 \mathrm{~d}$ of age and assigned randomly to each of the six experimental treatments (four rabbits/ treatment). Rabbits had ad libitum access to feed and freshwater over the trial, and received no antibiotic treatment. At $41 \mathrm{~d}$ of age $(1.13 \pm 0.005 \mathrm{~kg}$ body weight $)$ all rabbits were fitted with plastic collars $1 \mathrm{~h}$ in the morning from 9:00 to 10:00 $\mathrm{h}$ (maximum) to collect the soft faeces to be used as inoculum for the in vitro incubations. The soft faeces were wrapped in aluminium foil to reduce air exposure, and were immediately transported to the laboratory into thermal flasks. Soft faeces were used as inoculum for in vitro caecal fermentations because a previous study by our group (Abad-Guamán et al. 2018) demonstrated that caecal and soft faeces inocula resulted in similar gas production parameters for the same substrates used in the present study, and this procedure avoids the slaughter of rabbits.

\subsection{Substrates and in vitro procedure}

Five fibrous (or derived from fibrous) ingredients were selected as substrates for the in vitro incubations: CEL (NPC Cello-Oligo, Nippon Paper Industries Co., Tokyo, Japan; according to the manufacturer contained $96.6 \%$ cellobiose $\beta 1-4,1.9 \%$ cello-oligosaccharide, and 1.5\% glucose), D-(+)-glucose (GLU, Sigma n. 8270), sugar beet pulp (SBP, Fipec, Nordic Sugar, Copenhagen, Denmark; contained 646 g total dietary fibre (TDF) and $369 \mathrm{~g}$ neutral detergent fibre (NDF)/ $\mathrm{kg}$ DM; both values corrected for ash and crude protein), SBP pectin (PEC, Betapec RU 301, Herbstreith \& Fox, Neuenbürg, Germany), and wheat straw (WS, Pagran, PITE S.A., Tordesillas, Spain; contained $785 \mathrm{~g}$ TDF and $748 \mathrm{~g} \mathrm{NDF} / \mathrm{kg} \mathrm{DM})$. All substrates have low content in starch and protein, and were expected to have a wide range of fermentation rate and extent. Thus, most chemical constituents of CEL, SBP, PEC and WS cannot be digested by endogenous enzymes and absorbed by rabbits, but GLU is a rapid and completely fermentable substrate. 
Table 1. Ingredients and chemical composition of the experimental diets fed to donor rabbits.

\begin{tabular}{|c|c|c|}
\hline & \multicolumn{2}{|c|}{ Experimental diets } \\
\hline & Low soluble fibre & High soluble fibre \\
\hline \multicolumn{3}{|l|}{ Ingredients [ $\mathrm{g} / \mathrm{kg}$ as fed] } \\
\hline Dehydrated alfalfa & 150 & 150 \\
\hline Soybean meal & 80.0 & 80.0 \\
\hline Wheat & 227 & 217 \\
\hline Wheat bran & 280 & 129.7 \\
\hline Wheat straw & 100 & 50.0 \\
\hline Beet pulp & 0.00 & 180 \\
\hline Sunflower meal & 99.7 & 130 \\
\hline High oleic sunflower oil & 8.50 & 8.50 \\
\hline Sunflower oil & 21.5 & 21.5 \\
\hline L-Lysine & 4.40 & 4.40 \\
\hline DL-Methionine & 0.80 & 0.60 \\
\hline L-Threonine & 3.10 & 3.20 \\
\hline Calcium carbonate & 12.0 & 7.00 \\
\hline Sodium chloride & 3.00 & 3.10 \\
\hline Calcium phosphate & 5.00 & 10.0 \\
\hline Vitamin/mineral premix* & 5.00 & 5.00 \\
\hline \multicolumn{3}{|c|}{ Chemical composition $[\mathrm{g} / \mathrm{kg} \mathrm{DM}]$} \\
\hline Dry matter & 908 & 908 \\
\hline Ash & 70.8 & 67.5 \\
\hline Crude protein & 169 & 168 \\
\hline Total dietary fibre ${ }^{\dagger}$ & 391 & 442 \\
\hline Soluble fibre ${ }^{\ddagger}$ & 84.0 & 130 \\
\hline Neutral detergent fibre ${ }^{\dagger}$ & 307 & 312 \\
\hline Acid detergent fibre & 165 & 185 \\
\hline Acid detergent lignin & 31.0 & 33.0 \\
\hline Starch & 226 & 182 \\
\hline Ether extract & 53.8 & 48.7 \\
\hline Sugars & 79.9 & 81.7 \\
\hline
\end{tabular}

*Mineral and vitamin composition (per kg of complete diet): $20 \mathrm{mg} \mathrm{Mn}$ as $\mathrm{MnO} ; 59.2 \mathrm{mg} \mathrm{Zn}$ as $\mathrm{ZnO} ; 10 \mathrm{mg} \mathrm{Cu}$ as $\mathrm{CuSO}_{4} \cdot 5 \mathrm{H}_{2} \mathrm{O} ; 1.25 \mathrm{mg} \mathrm{I}$ as Kl; $0.495 \mathrm{mg} \mathrm{Co}$ as $\mathrm{CoCO}_{3} \cdot \mathrm{H}_{2} \mathrm{O} ; 76 \mathrm{mg} \mathrm{Fe}$ as $\mathrm{FeCO}_{3} ; 8375$ Ul vitamin $\mathrm{A} ; 750$ UI vitamin $\mathrm{D}_{3}, 20$ UI vitamin $\mathrm{E}$ as DL-a-tocopherol acetate, $1.0 \mathrm{mg}$ vitamin $\mathrm{K} ; 1.0 \mathrm{mg}$ vitamin $\mathrm{B}_{1} ; 2 \mathrm{mg}$ vitamin $\mathrm{B}_{2} ; 1 \mathrm{mg}$ vitamin $\mathrm{B}_{6}$; $20 \mathrm{mg}$ niacin; $54.1 \mathrm{mg}$ betaine; $137,5 \mathrm{mg}$ choline chloride; $66 \mathrm{mg}$ of robenidine; $50 \mathrm{mg}$ of ethoxyquin, provided by Trouw Nutrition (Madrid, Spain); "Values were corrected for ash and crude protein; ${ }^{\ddagger}$ Calculated as TDF minus NDF; values corrected for ash.

Samples (250 mg DM) of each substrate were carefully weighed into 115-ml glass vials and were subjected to a two-step pepsin-pancreatin in vitro digestion (pre-digestion) following the method of Ramos et al. (1992), with the only exception that the contents of the vials were not filtrated at the end of the procedure. All vials were stored at $4^{\circ} \mathrm{C}$ overnight to stop the digestion process, and placed back in an incubator at $40^{\circ} \mathrm{C}$ for $1 \mathrm{~h}$ before starting the in vitro incubations (Ocasio-Vega et al. 2018a). A total of 120 vials with substrate $(3$ cellobiose concentrations $\times 2$ donors' diet $\times 5$ substrates $\times 4$ rabbits) and 24 vials without substrate (blanks; one per rabbit) were used.

Soft faeces from each rabbit were mixed with the culture medium of Goering and Van Soest (1970) in a proportion of $720 \mathrm{mg}$ soft faeces to $100 \mathrm{ml}$ medium and homogenised with a blender for $2 \mathrm{~min}$. The soft faeces/medium ratio was selected from previous studies by our group (unpublished results). Vials were filled up with $25 \mathrm{ml}$ of the mixture using a Watson-Marlow 520 UIP31 peristaltic pump (WatsonMarlow Fluid Technology Group, Cornwall, United Kingdom), sealed with rubber stoppers, and incubated at $40^{\circ} \mathrm{C}$ for $144 \mathrm{~h}$ to ensure that asymptote gas production 
was reached for all substrates. All procedures were carried out under continuous $\mathrm{CO}_{2}$ flushing at $40^{\circ} \mathrm{C}$. Gas production was measured at $4,6,9,12,20,24,30,35,48,58,72$, 96 and 120 and $144 \mathrm{~h}$ using a pressure transducer (Delta Ohm DTP704-2BGI, Herter Instruments SL, Barcelona, Spain) and a plastic syringe, the gas produced at each measurement time being released. Immediately after measuring the gas production at $24 \mathrm{~h}, 1 \mathrm{ml}$ of each vial content was taken using an insulin syringe, mixed with $20 \mu \mathrm{l}$ of $\mathrm{H}_{2} \mathrm{SO}_{4}(10 \% \mathrm{vol} / \mathrm{vol})$ and stored at $-20^{\circ} \mathrm{C}$ for VFA analysis.

\subsection{Chemical analyses}

The procedures of the AOAC (2000) were used to determine DM (method 934.01), ash (method 942.05), crude protein (method 968.06), ether extract (method 920.39), starch (amyloglucosidase- $\alpha$-amylase method; method 996.11), TDF (method 985.29) and acid detergent fibre (method 973.187) content in the experimental diets. Dietary NDF was determined using the filter bag system (Ankom Technology, New York) according to Mertens (2002), and a thermo-stable amylase without any sodium sulphite added. Data were corrected for ash and protein content, as indicated for TDF. Dietary acid detergent lignin content was analysed according to Goering and Van Soest (1970). The soluble fibre content was calculated by difference as TDF-NDF.

Samples for VFA analysis were thawed, centrifuged $\left(13,000 \mathrm{~g}, 15 \mathrm{~min}, 4^{\circ} \mathrm{C}\right), 0.8 \mathrm{ml}$ of the supernatant were mixed with $0.5 \mathrm{ml}$ of deproteinising solution $(0.06 \%$ of crotonic acid and $2 \%$ of metaphosphoric; in volume) and stored overnight at $4{ }^{\circ} \mathrm{C}$. Samples were centrifuged again and the supernatant was transferred to chromatography vials. Samples were stored at $-20^{\circ} \mathrm{C}$ until analysis by gas chromatography (Carro et al. 1992) in a Perkin Elmer Autosystem XL gas chromatograph (PerkinElmer Inc., Shelton, CT, USA) with an automatic injector, detector flame ionisation and a semicapillary column TR-FFAP $30 \mathrm{~m} \times 0.53 \mathrm{~mm} \times 1 \mu \mathrm{m}$ (Supelco, Barcelona, Spain).

\subsection{Calculations and statistical analysis}

Gas production values measured at each time and VFA values at $24 \mathrm{~h}$ were corrected for the amount of gas and VFA, respectively, produced in the corresponding blanks to correct for endogenous production (Ocasio-Vega et al. 2018a). Gas production data were fitted to the logistic model described by Schofield et al. (1994):

$$
\text { Gas production }=V_{\mathrm{f}} /\left[1+e^{[2-4 k(t-\mathrm{Lag})]}\right] \text {, }
$$

where $V_{\mathrm{f}}$ is the final asymptotic gas production, $k$ is the fractional rate of gas production, Lag is the initial delay in the onset of gas production and $t$ is the time of gas measurement. The $V_{\mathrm{f}}, k$ and Lag parameters were estimated by an iterative least squares procedure (Marquardt algorithm) using the NLIN procedure of SAS (version 9.2; SAS Inst. Inc., Cary, NC, USA). The maximum gas production rate $\left(\mu_{\mathrm{m}}\right)$ and the time when $\mu_{\mathrm{m}}$ is reached $\left(T_{\mathrm{i}}\right)$ were calculated according to Schofield et al. (1994) as

$$
\mu_{\mathrm{m}}=k \times V_{\mathrm{f}}, \text { and } T_{\mathrm{i}}=\operatorname{Lag}+\left(V_{f} /\left(2 \times \mu_{\mathrm{m}}\right)\right) .
$$


Data on gas production parameters and VFA production were analysed as a mixed model including the cellobiose dose $(0.00,7.50$ and $15.0 \mathrm{~g} / \mathrm{l})$, diet of donor rabbits (LSF and HSF), substrate and their respective interactions as fixed effects, and donor rabbits (inoculum) as a random effect using the PROC MIXED of the SAS package (SAS Inst. Inc., Cary, NC). When the effect of cellobiose supplementation or its interaction either with donor's diet or with substrate was significant, linear and quadratic polynomial contrasts were used to test the linear and quadratic effects of cellobiose level and its interaction with the level of soluble fibre or substrate. When a significant effect of cellobiose supplementation, substrate or any interaction was detected $(p<0.05)$, the Tukey test was used for mean comparisons. Significance was declared at $p<0.05$, and $p$ values between 0.05 and 0.10 were considered to be a trend. Relationships between total VFA production profile determined in vitro and in vivo were tested by correlation analyses using the CORR procedure of SAS (SAS Inst. Inc., Cary, NC). As previously stated, values of total VFA concentration and VFA profile in the caecum of rabbits receiving the same experimental treatments have been reported by Ocasio-Vega et al. (2018b).

\section{Results}

Table 2 indicates the influence of cellobiose supplementation and donors' diet on gas production parameters and VFA production in the cultures without substrate (blanks). There were no cellobiose $\times$ donors' diet interactions with the exception of a trend for $V_{\mathrm{f}}$ $(p=0.086)$ and isovalerate proportion $(p=0.054)$. Values of $V_{\mathrm{f}}, k$ and $T_{\mathrm{i}}$ were quadratically influenced by cellobiose supplementation $(p=0.003,0.002$ and 0.001 , respectively), with blanks from rabbits receiving the 7.5 dose of cellobiose having lower $k$ values $(p<0.05)$ and greater $T_{\mathrm{i}}$ values $(p<0.05)$ than those from rabbits receiving the 0 and 15 doses $(0.090,0.072$ and $0.094 \% / h$ for $0,7.5$ and 15 doses, respectively, and $5.70,6.99$ and $5.44 \mathrm{~h}$, respectively; values averaged across diets). There were no effects of donors' diet on any gas production parameter $(p=0.31-1.00)$.

Total VFA production in the blanks increased linearly $(p=0.001)$ by cellobiose supplementation, with no differences between the 7.5 and 15 doses $(5.92,9.28$ and $9.71 \mathrm{mmol} / \mathrm{l}$ for $0,7.5$ and 15 doses, respectively; values averaged across diets). In contrast, cellobiose supplementation did not affect VFA profile, with the exception of the molar proportion of butyrate $(p=0.036)$, which was greater $(p<0.05)$ for the 7.5 dose than for the 0 dose with both donors' diets. Total VFA production was greater for LSF compared with the HSF inoculum (9.11 vs. $7.49 \mathrm{mmol} / \mathrm{l} ; p=0.035)$, but donors' diet had no influence on VFA profile in the blanks $(p=0.18-0.75)$.

Table 3 and Figures 1 and 2 indicate the influence of cellobiose supplementation and donors' diet on gas production kinetics of the incubated substrates. As expected, gas production parameters differed widely $(p<0.001)$ among substrates. There were also marked differences among substrates in the response to experimental factors, which lead to cellobiose $\times$ donors' diet $\times$ substrate interactions $(p \leq 0.003)$ for $k$, lag and $\mu_{\mathrm{m}}$. As indicated in Figure 1, gas production kinetics of SBP and WS were not modified either by cellobiose supplementation or donor's diet, but some effects were observed for the rest of substrates, being those more pronounced for CEL than for GLU and PEC.

Cellobiose $\times$ substrate interactions were only observed for Lag $(p=0.005)$, as cellobiose supplementation decreased linearly Lag values for CEL and PEC ( $p=0.003$ and 0.012 , 


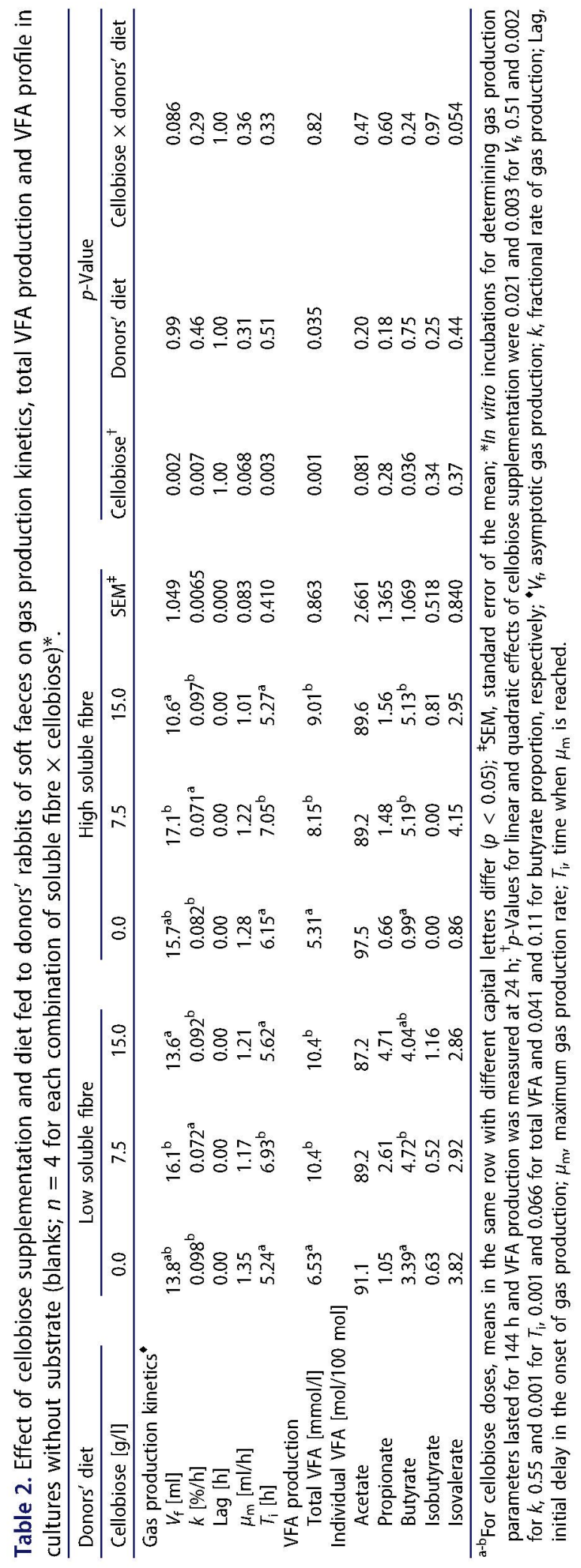


Table 3. Effects of cellobiose supplementation and diet fed to donors' rabbits of soft faeces on gas production kinetics of different substrates in $144 \mathrm{~h}$ in vitro incubations ( $n=4$ for each combination of soluble fibre $x$ cellobiose).

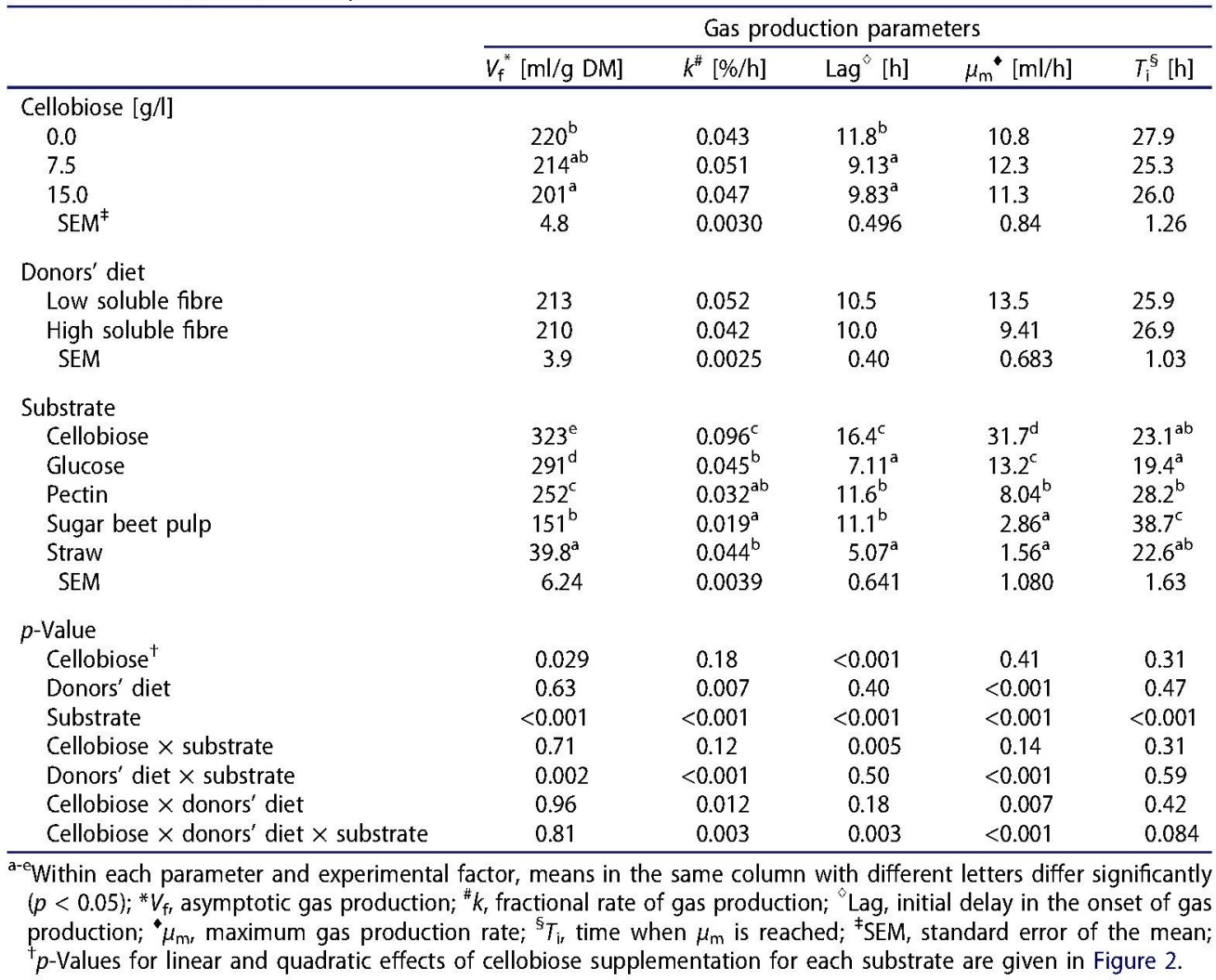

respectively) but had no effect for the rest of substrates (Figure 2(c)). Donors' diet $\times$ substrate interactions $(p \leq 0.002)$ were found for $V_{\mathrm{f}}, k$ and $\mu_{\mathrm{m}}$. The $V_{\mathrm{f}}$ values for CEL substrate were greater with the LSF inoculum than with HSF one, but the opposite was observed for PEC (Figure 2(a)), and no effects were detected for GLU, SBP and WS. Both $k$ and $\mu_{\mathrm{m}}$ values for CEL were greater with the LSF compared with HSF inoculum $(p<0.001 ; 0.119 v s .0 .073 \% / \mathrm{h}$, and $41.5 v$ s. $21.9 \mathrm{~h}$, respectively), but there were no effects of donors' diet on $k$ and $\mu_{\mathrm{m}}$ values for the rest of substrates.

As previously indicated, CEL was the most affected substrate by the experimental treatments, and cellobiose $\times$ donors' diet interactions were detected for $k$, Lag, $\mu_{\mathrm{m}}$ and $T_{\mathrm{i}}$ values of CEL accounting for the triple interaction cellobiose $\times$ donors' diet $\times$ substrate $(p \leq 0.084$. Table 3). Whereas $k$ and $\mu_{\mathrm{m}}$ were increased linearly and Lag and $T_{\mathrm{i}}$ decreased linearly by cellobiose supplementation with the HSF inoculum $(p=0.014,0.042,0.001$ and $<0.001$, respectively; Figure 2(b-e)), with the LSF inoculum cellobiose supplementation only affected $k$ values ( $p=0.043$ ) of CEL, which were quadratically increased and had maximal values for the 7.5 dose (Figure 2(b)). The incubation of CEL with the HSF0 inoculum resulted in higher Lag and $T_{\mathrm{i}}$ values compared with the HSF7.5 and HSF15 inocula, without differences between the 7.5 and 15 doses of cellobiose. 
$0.0 \mathrm{~g}$ of cellobiose $\mathrm{L}$

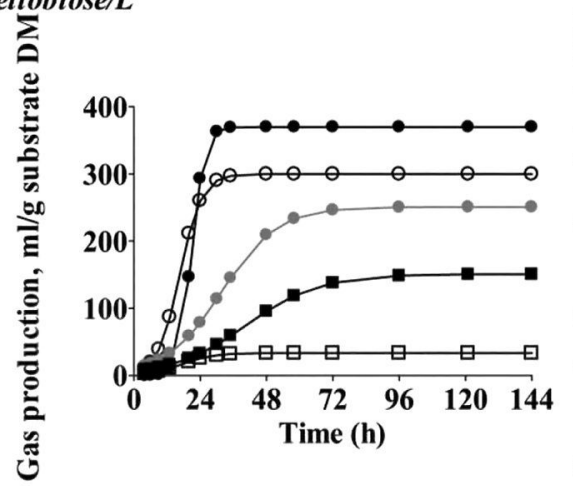

$7.5 \mathrm{~g}$ of cellobiose/L

立

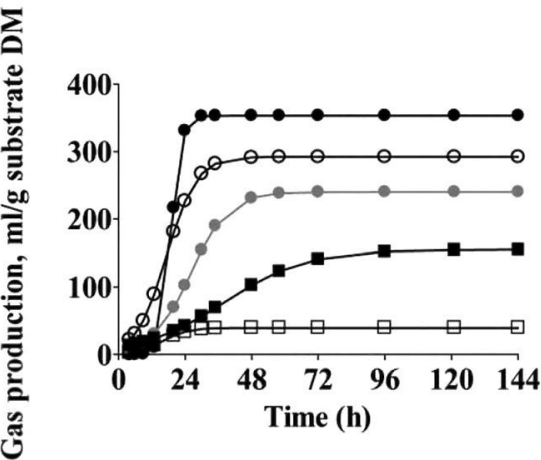

$15.0 \mathrm{~g}$ of cellobiose $\mathrm{L}$

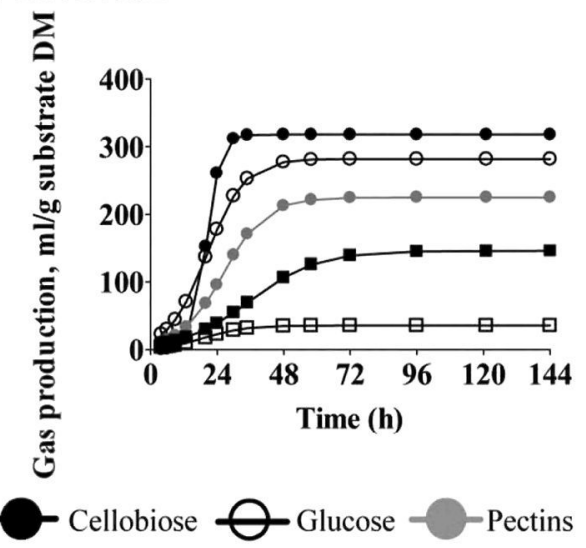

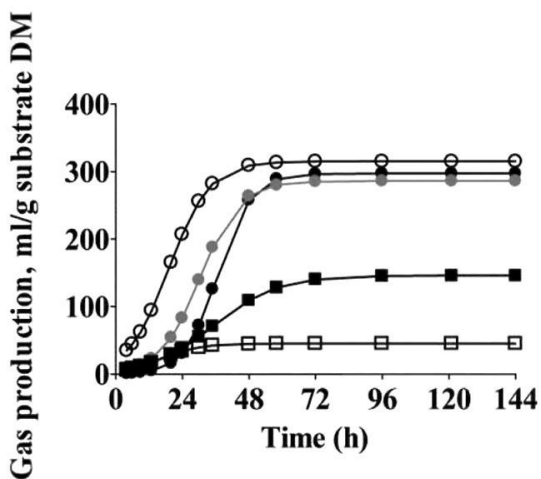
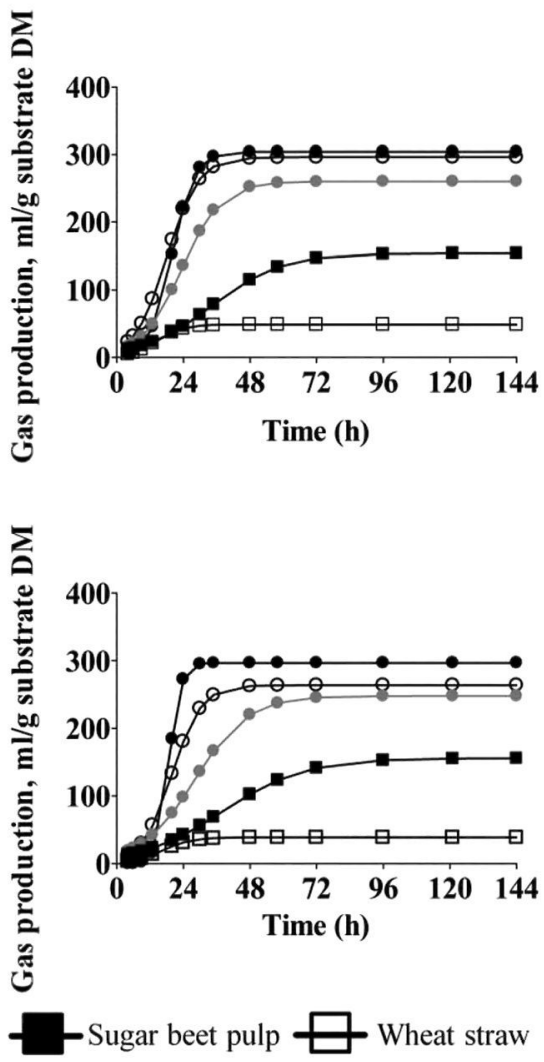

Figure 1. Effect of cellobiose supplementation $(0.0,7.5$ and $15 \mathrm{~g} / \mathrm{l})$, diet fed to donors' rabbits of soft faeces (LSF or HSF) and incubated substrate on gas production kinetics in $144 \mathrm{~h}$ in vitro incubations $(n=4$; Standard error $=10.1 \mathrm{ml} / \mathrm{g}$ DM substrate) .

Cellobiose $\times$ donors' diet interactions were only detected for $k$ and Lag parameters for GLU, and for Lag for PEC. When both substrates were incubated with the HSF inoculum, cellobiose supplementation increased linearly Lag values for GLU ( $p=0.006)$ and decreased linearly Lag values for $\operatorname{PEC}(p=0.001)$. 
A)

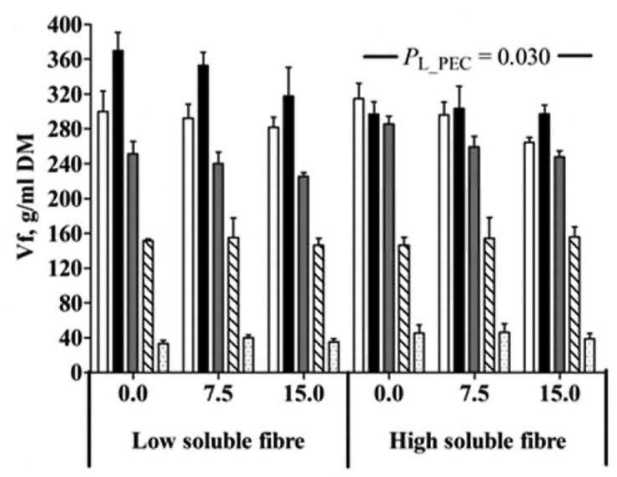

C)

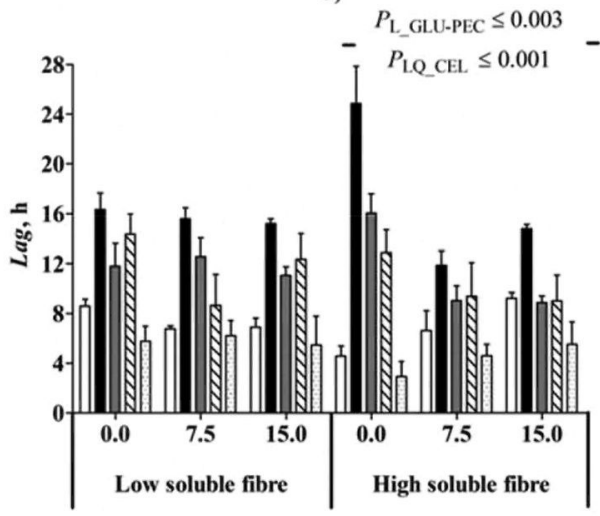

E)

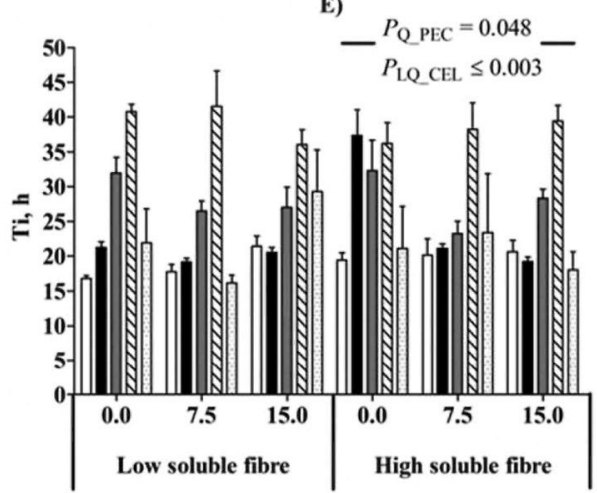

B)

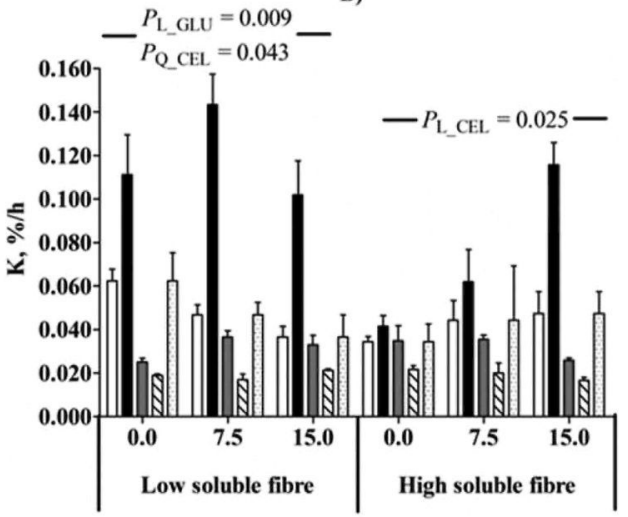

D)

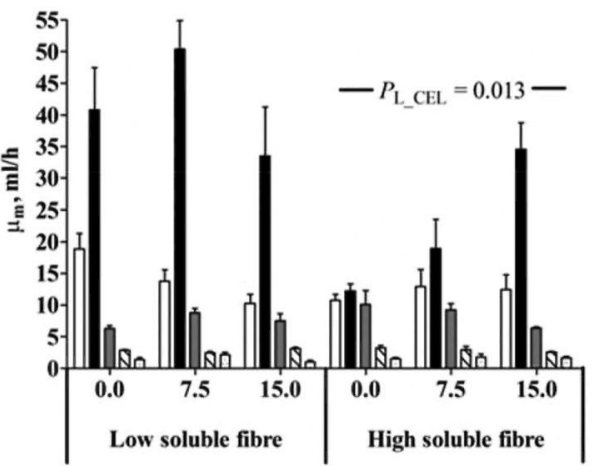

Figure 2. Effect of cellobiose supplementation, diet fed to donors' rabbits of soft faeces (LSF or HSF) and incubated substrate (glucose [GLU], cellobiose [CEL], SBP pectins (Pectins [PEC]), SBP, and WS) on gas production parameters in $144 \mathrm{~h}$ in vitro incubations ( $n=4$ rabbits/treatment). (a) $V_{f}$ : asymptotic gas production; (b) $k$ : fractional rate of gas production; (c) Lag: initial delay in the onset of gas production; (d) $\mu_{\mathrm{m}}$ : maximum gas production rate; (e) $T_{\mathrm{i}}$ : time when $\mu_{\mathrm{m}}$ is reached. Within each diet (LSF or HSF) and for each substrate, $P_{\mathrm{L}}$ and $P_{\mathrm{Q}}$ indicate linear and quadratic effects $(p<0.05)$ of cellobiose, respectively. Bars indicate the standard error. 
Values of gas and VFA production at $24 \mathrm{~h}$ of incubation are indicated in Table 4 and Figure 3. Gas production at $24 \mathrm{~h}$ for GLU, PEC and SBP substrates was not affected either by the dose of cellobiose or by donors' diet, but significant effects of both factors were detected for CEL, thus leading to interactions among the experimental treatments. Cellobiose $\times$ donors' diet interactions for the 24 -h gas production were only detected for CEL and WS substrates ( $p=0.002$ and 0.009 , respectively). For CEL, cellobiose supplementation increased linearly gas production at $24 \mathrm{~h}$ with the HSF inoculum $(p<0.001 ; 43.0,213$ and $253 \mathrm{ml} / \mathrm{g}$ DM for 0, 7.5 and 15 doses, respectively; Figure 3(a)), but had no effect with the LSF inoculum. For WS, cellobiose supplementation increased quadratically 24 -h gas production with the LSF inoculum $(p=0.001 ; 24.3,35.6$ and $19.2 \mathrm{ml} / \mathrm{g}$ DM for 0, 7.5 and 15 doses, respectively; Figure 3(a)), but had no effect with the HSF inoculum. Donors' diet only affected the 24-h gas production for CEL substrate, being greater with the LSF than with the HSF inoculum (289vs. $170 \mathrm{mmol} / \mathrm{l} ; p<0.001)$.

Interactions cellobiose $\times$ substrate $(p=0.001)$ and donors' diet $\times \operatorname{substrate}(p=0.006)$ were observed for total VFA production (Table 4). Whereas no effects of cellobiose or donors' diet were detected for GLU and SBP, cellobiose supplementation increased total VFA for CEL and PEC with both inocula ( $p=0.018$, quadratic for CEL; $p=0.034$ and 0.016 , linear and quadratic for PEC), and decreased VFA production for WS with the HSF inoculum $(p=0.005$, linear; Figure $3(\mathrm{~b}))$. Total VFA production for CEL was greater with LSF than with HSF inoculum (20.7 vs. $12.9 \mathrm{mmol} / 1 ; p=0.014)$, but the opposite was found for WS (3.97vs. $6.21 \mathrm{mmol} / \mathrm{l} ; p=0.005)$.

The VFA profile differed widely among substrates, with CEL having the lowest acetate $(p<0.05)$ and greatest butyrate $(p<0.05)$ proportions. Neither valerate nor caproate were detected in any sample, and no propionate, isobutyrate and isovalerate were detected in the fermentation of GLU. No effects of either cellobiose supplementation or donors' diet on VFA profile were observed for GLU, PEC, SBP and WS substrates (Figure 3). In contrast, for CEL substrate cellobiose supplementation decreased isovalerate proportions with the HSF inoculum $(p=0.001$, linear and 0.018 , quadratic), but had no effect with the LSF inoculum. Donors' diet only affected VFA profile for CEL substrate, with HSF-inoculated cultures having greater acetate and isovalerate proportions $(81.2$ vs. $55.6 \mathrm{~mol} / 100 \mathrm{~mol}, p=0.001$, and 1.04 vs. $0.07 \mathrm{~mol} /$ $100 \mathrm{~mol}, p=0.026$, respectively) and lower butyrate proportions (13.6 vs. $42.6 \mathrm{~mol} /$ $100 \mathrm{~mol}, p<0.001$ ) than those LSF-inoculated.

There were no relationships between either total VFA concentrations or VFA profile measured in the caecum of rabbits receiving the same experimental treatments (values reported by Ocasio-Vega et al. 2018b) and the values measured in vitro in the present study for CEL, GLU, SBP and PEC substrates. However, a positive relationship $(r=0.90 ; p=0.015 ; n=6)$ was observed between total VFA caecal concentrations in vivo and in vitro when WS was used as substrate.

\section{Discussion}

In agreement with previous results in pigs and ruminants demonstrating the influence of the diet of donors on in vitro fermentation of different substrates (Bindelle et al. 2007; Mateos et al. 2013), the production of both gas and VFA in the blanks was 
A)

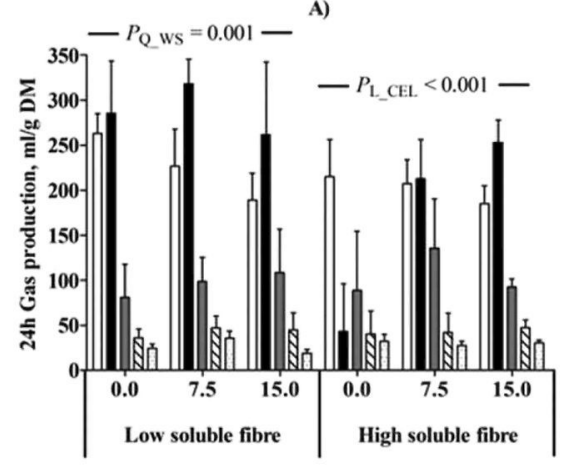

C)

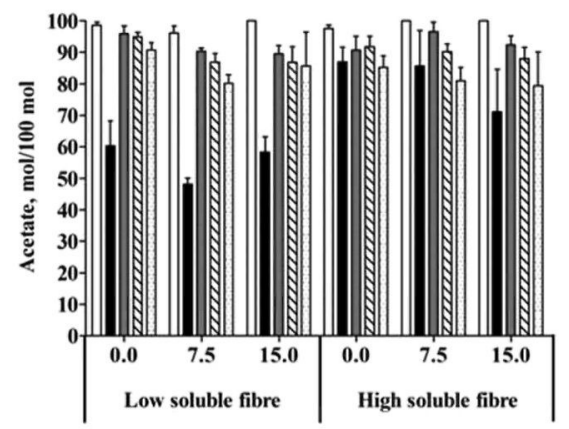

E)

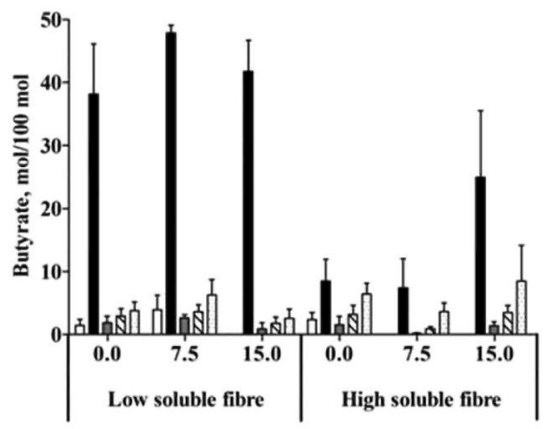

G)

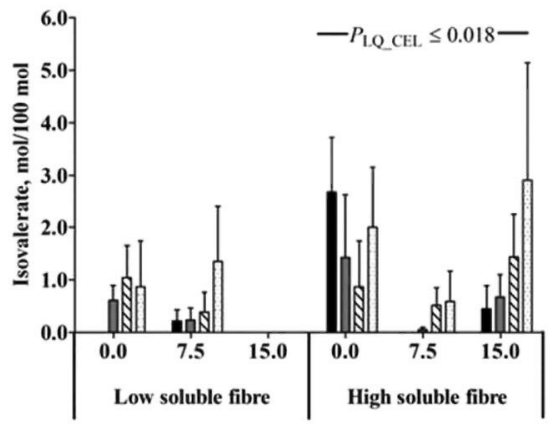

B)

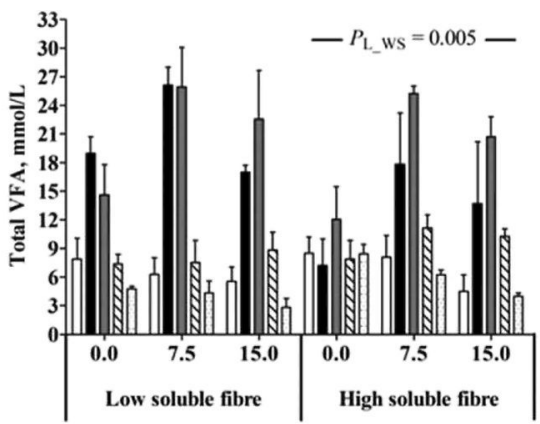

D)

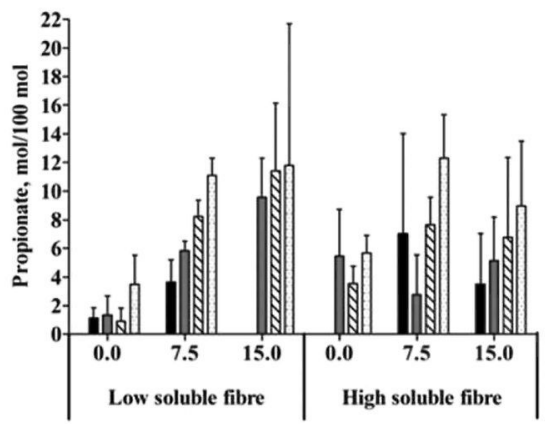

F)

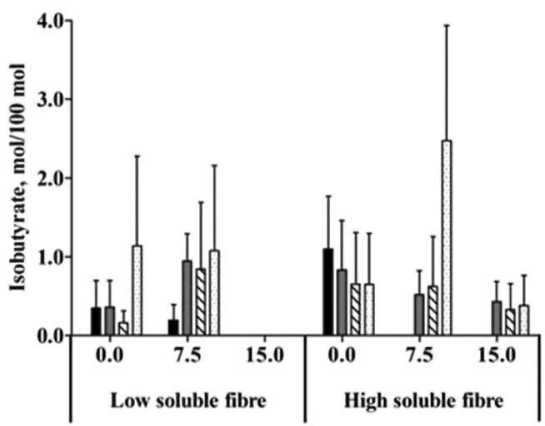

Figure 3. Effect of cellobiose supplementation, fed to donors' rabbits of soft faeces (LSF or HSF) and incubated substrate (glucose [GLU], cellobiose [CEL], SBP pectins (Pectins [PEC]), SBP, and WS) on gas and total volatile fatty (VFA) production and molar proportions of individual VFA after $24 \mathrm{~h}$ in vitro incubation ( $n=4$ rabbits/treatment). Within each diet (LSF or HSF) and for each substrate, $P_{\mathrm{L}}$ and $P_{\mathrm{Q}}$ indicate linear and quadratic effects $(p<0.05)$ of cellobiose, respectively. Bars indicate the standard error. 


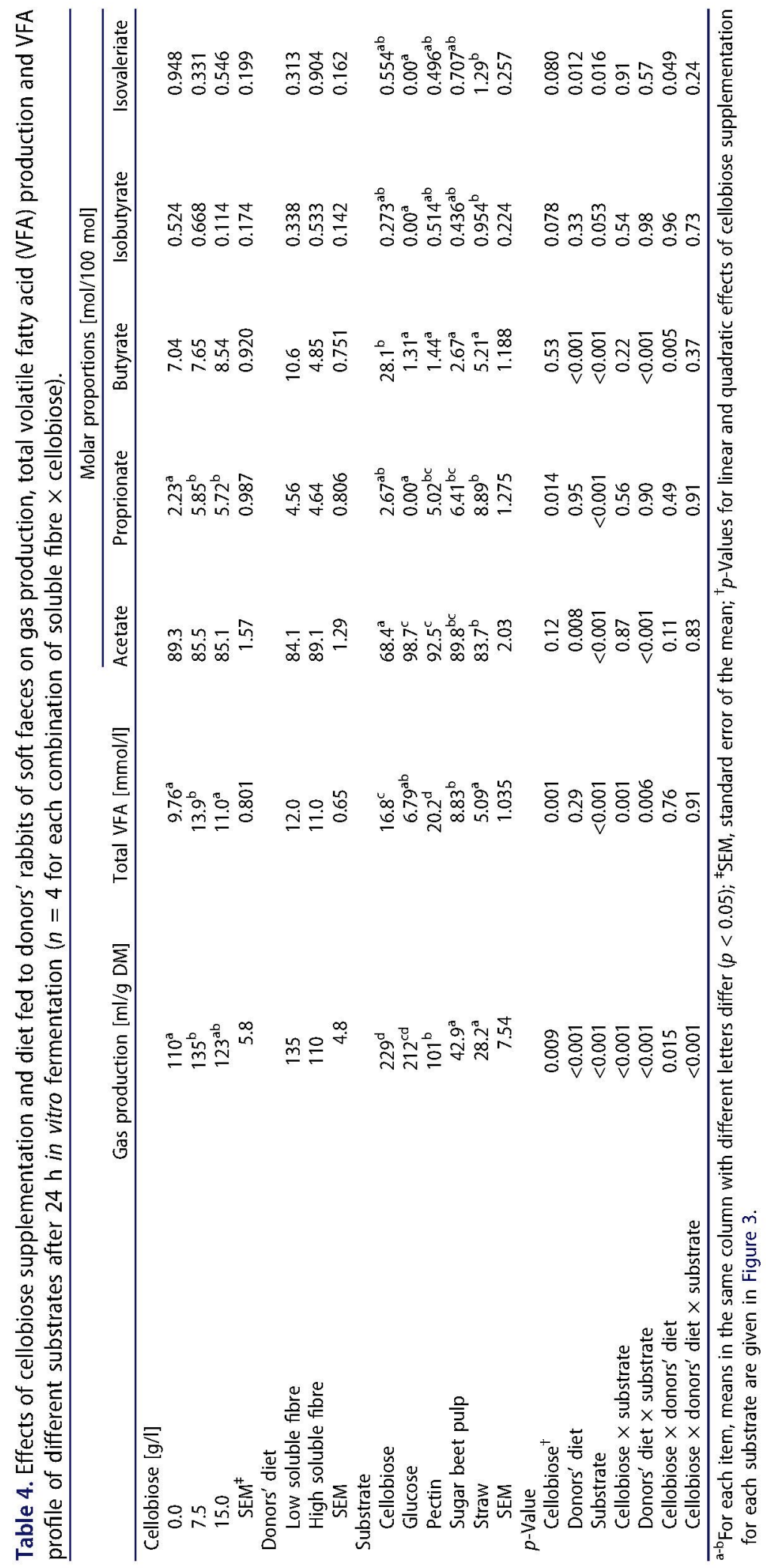


strongly influenced by the cellobiose supplementation. In contrast, there was no effect of the soluble fibre content in the diet, and no cellobiose $x$ donors' diet interaction was detected. The lack of influence of the soluble fibre content on blanks fermentation was unexpected, especially taking into account the positive influence exerted by the inclusion of soluble fibre in the diet on fibre digestibility, both at ileal and faecal level, observed previously by our group (Abad-Guamán et al. 2015). Moreover, the increase of dietary soluble fibre increased the in vitro gas production at $18 \mathrm{~h}$ when the indigestible residue of the two-step in vitro digestibility of the diet was used as substrate (Rodríguez-Romero et al. 2011).

However, donors' diet affected both gas production parameters and VFA profile when substrates were incubated (Tables 3 and 4 ). The significant cellobiose $\times$ donors' diet interactions detected for several parameters suggest differences in the fermentative activity of LSF and HSF inocula, which might be related to different microbial communities in both inocula. In fact, Gómez-Conde et al. (2009) observed differences in caecal microbiota diversity in rabbits fed diets with soluble fibre contents $(79-131 \mathrm{~g}$ soluble fibre $/ \mathrm{kg} \mathrm{DM}$ ) similar to those used in our study. Furthermore, the increase of soluble fibre using SBP reduced the biodiversity of caecal microbiota (RodríguezRomero et al. 2012), probably due to the highly specialised bacterial community required to degrade its pectin content.

The CEL was the most affected substrate by both cellobiose supplementation and donors' diet. The combination of HSF inoculum with no cellobiose supplementation increased Lag value for CEL, indicating that its fermentation started later. This could be due to the lack of adaptation of the caecal microbiota to cellobiose fermentation in rabbits fed HSF diet and no cellobiose during the $7 \mathrm{~d}$ of diet adaptation before conducting the in vitro trial. However, the inoculum from rabbits fed LSF diet with no cellobiose adapted better to CEL fermentation. In addition, total VFA was quadratically increased by cellobiose supplementation with both inocula, with values for the 7.5 dose $(22.0 \mathrm{mmol} / \mathrm{l})$ being greater than those for the 0 and 15 doses (13.1 and $15.3 \mathrm{mmol} / \mathrm{l}$, respectively). Although the differences did not reach the significance level, acetate and butyrate proportions were numerically lower and greater, respectively, for the 7.5 dose compared with the 0 and 15 doses with the LSF inoculum (48.1 vs. 60.4, and $58.3 \mathrm{~mol} / 100 \mathrm{~mol}$ for acetate, and 47.8 vs. 38.2 and $41.7 \mathrm{~mol} / 100 \mathrm{~mol}$ for butyrate, respectively). The fermentation of CEL with the LSF inoculum generated more total VFA than with the HSF inoculum (20.7 vs. $12.9 \mathrm{mmol} / \mathrm{l})$, and increased butyrate $(42.6$ vs. $13.6 \mathrm{~mol} / 100 \mathrm{~mol}$ ) at expenses of acetate $(55.6$ vs. $81.2 \mathrm{~mol} / 100 \mathrm{~mol})$. These results would help to explain the positive effect of the LSF7.5 treatment on rabbit's health observed by Ocasio-Vega et al. (2018b) using the same cellobiose's doses, who reported that rabbits from the LSF7.5 treatment had lower mortality between 34 and $61 \mathrm{~d}$ of age than those from LSF0 and LSF15 treatments (8.57 vs. 25.7 and 17.1\%, respectively) and these results were confirmed by Ocasio-Vega (2018b). In contrast, supplying increasing doses of cellobiose to HSF-fed rabbits linearly increased their mortality (5.71, 14.3 and $22.9 \%$ for $0,7.5$ and 15 doses, respectively). The two groups with the lowest mortality (LSF7.5 and HSF0) had also greater proportions of butyrate in the ileal digesta $(2.44$ and $1.89 \mathrm{~mol} / 100 \mathrm{~mol}$ for LSF7.5 and HSF0, respectively; values lower than $1.50 \mathrm{~mol} /$ $100 \mathrm{~mol}$ for the rest of treatments. Ocasio-Vega et al. (2018b), and this could be related 
to the positive effects of butyrate on intestinal health reported in numerous studies (Guilloteau et al. 2010; Cushing et al. 2015).

However, our in vitro results cannot explain the negative effects of cellobiose supplementation on mortality of rabbits fed the HSF diet observed by Ocasio-Vega et al. (2018b). It might be only hypothesised a potential negative effect of cellobiose on the intestinal microbiota of HSF-fed rabbits, or an unknown mechanism explaining the low mortality found with both HSF0 and LSF7.5 treatments. However, the in vitro fermentation profile of CEL with HSF0 and LSF7.5 inocula differed widely, both quantitatively (7.23 vs. $26.1 \mathrm{mmol}$ total VFA/L) and qualitatively (for acetate $87.0 \mathrm{vs}$. $48.1 \mathrm{~mol} / 100 \mathrm{ml}$ ) and butyrate proportions $(8.47$ vs. $47.8 \mathrm{~mol} / 100 \mathrm{ml})$. It should be noted that our in vitro study was conducted using soft faeces as inoculum and the possible effects of cellobiose supplementation on other parts of the digestive tract could not be assessed. Therefore, it would be interesting to analyse whether the use of inoculum from the small intestine, where at least part of CEL can be fermented, produces similar results to those observed in this study for soft faeces or not.

Yang et al. (2010) found no differences in gas and total VFA production between GLU and CEL when both were fermented with fresh caecal content, but in our study CEL fermentation resulted in greater $V_{\mathrm{f}}, k$ and $\mu_{\mathrm{m}}$ values and total VFA production compared with GLU. The fermentation of CEL generated lower acetate and greater butyrate proportions than that of GLU, which is in agreement with the results of Yang et al. (2010). This fermentation pattern of GLU compared with that of oligodextrans has been observed in humans using batch cultures inoculated with slurried faecal bacteria (Olano-Martín et al., 2000). Van Zanten et al. (2012) reported that CEL stimulated the growth of Lactobacillus acidophilus NCFM and Bifidobacterium animalis subsp. lactis Bl-04 under laboratory conditions, and the supply of CEL with this two bacteria to an in vitro colonic model increased butyrate proportions and reduced the Bacteroidetes/ Firmicutes ratio, whose high values have been linked with obesity and type 2 diabetes in humans. In farm animals, Jiao et al. (2014) observed that supplementing cellooligosaccharide $(1.5-4.5 \mathrm{~g} / \mathrm{kg}$ diet) to weaned pigs increased Lactobacillus spp. and decreased Clostridium concentrations in jejunal contents, and Song et al. (2013) reported that cello-oligosaccharide supplementation to broilers increased viable counts of Lactobacillus spp. in caecal contents and decreased the counts of Escherichia coli. These results indicate that CEL supplementation can modify the intestinal microbiota and produce shifts in VFA profile.

Although PEC is a highly fermentable substrate, its in vitro fermentation was less affected by the experimental factors than that of CEL. The linear reduction of Lag produced by cellobiose supplementation was also observed for CEL and might indicate the existence of a microbiota more adapted to ferment this substrate. The increased total VFA production observed in the cultures inoculated with soft faeces from cellobiose-supplemented rabbits is in accordance with this hypothesis. In contrast, the experimental factors did not affect SBP fermentation, and only affected total VFA production for WS. Both SBP and WS were the most complex substrates of all incubated, and this might be related to the lower effects of the experimental treatments detected for them.

Although the experimental factors influenced the fermentation of some substrates in our study, only a significant relationship between total VFA caecal concentrations in 
vivo and in vitro was observed when WS was used as substrate (not shown). The different conditions in rabbits caecum and in vitro (i.e. buffer capacity of the incubation medium, ratio substrate/incubation medium, digesta retention time, movements, etc.) can explain the lack of significant in vivo-in vitro relationships. It should be also considered that VFA are removed from the caecum by absorption and flow to the colon (Vernay 1987), whereas there is no absorption or digesta flow in the in vitro cultures. In addition, pure substrates were incubated in vitro, whereas non-digested fractions of feeds are the materials potentially fermented in the caecum. The conditions in the in vitro cultures may have caused a selection of some bacterial strains, as it has been previously demonstrated in in vitro cultures inoculated with fresh faeces from pigs (Boudry et al. 2012) and with ruminal fluid (Mateos et al. 2015).

In conclusion, cellobiose supplementation and donors' diet of rabbits did not affect the in vitro caecal fermentation of SBP and had only subtle effects on the fermentation of WS and GLU. In contrast, CEL fermentation was markedly affected by both factors, thus indicating changes in the fermentative activity of caecal digesta. Cellobiose supplementation to HSF-fed rabbits increased the rate of CEL fermentation for both tested doses, but in LSF-fed rabbits this effect was only observed for the 7.5 dose. These results were confirmed at $24 \mathrm{~h}$ of incubation, both in gas and VFA production. The inoculum of LSF-fed rabbits receiving the 7.5 cellobiose dose also resulted in the lowest acetate and greatest butyrate proportions compared with the rest of treatments, which might be related to the beneficial effects of this treatment on rabbits health observed in a previous study. These results might indicate that the dose of cellobiose required to exert a beneficial effect on rabbits caecal fermentation depends on the content of soluble fibre in the diet.

\section{Disclosure statement}

No potential conflict of interest was reported by the authors.

\section{Funding}

Funding from the Ministerio de Ciencia e innovación (Projects AGL2011-23885 and AGL201122628) and the Comunidad Autónoma de Madrid (CAM; Project MEDGAN ABI-2913) is gratefully acknowledged.

\section{ORCID}

César Ocasio-Vega (D) http://orcid.org/0000-0001-8987-1096

Rodrigo Abad-Guamán (B) http://orcid.org/0000-0002-2015-8548

Rebeca Delgado (D) http://orcid.org/0000-0002-8887-417X

Rosa Carabaño (i) http://orcid.org/0000-0003-0773-3920

María Dolores Carro (D) http://orcid.org/0000-0002-4221-9057

Javier García (D) http://orcid.org/0000-0003-2053-9225 


\section{References}

Abad-Guamán R, Carabaño R, Gómez-Conde MS, García J. 2015. Effect of type of fiber, site of fermentation, and method of analysis on digestibility of soluble and insoluble fiber in rabbits. $\mathbf{J}$ Anim Sci. 93:2860-2871.

Abad-Guamán R, Larrea-Dávalos JA, Carabaño R, García J, Carro MD. Forthcoming 2018. Influence of type of inoculum (ileal, caecal and faecal) on the in vitro fermentation 2 of different sources of carbohydrates in rabbits. World Rabbit Sci.

AOAC. 2000. Official methods of analysis of AOAC International. Arlington (VA): AOAC.

Bindelle J, Buldgen A, Lambotte D, Wavreille J, Leterme P. 2007. Effect of pig faecal donor and of pig diet composition on in vitro fermentation of sugar beet pulp. Anim Feed Sci Technol. 132:212-226.

Boletín Oficial del Estado. 2013. Real Decreto 53/2013. In: Normas básicas aplicables para la protección de los animales utilizados en experimentación y otros fines científicos, incluyendo la docencia. B.O.E. 34. p. 11370-11421.

Boudry C, Poelaert C, Portetelle D, Thewis A, Bindelle J. 2012. Discrepancies in microbiota composition along the pig gastrointestinal tract between in vivo observations and an in vitro batch fermentation model. J Anim Sci. 90:393-396.

Carro MD, Lebzien P, Rohr K. 1992. Influence of yeast culture on the in vitro fermentation (Rusitec) of diets containing variable portions of concentrates. Anim Feed Sci And Technol. $37: 209-220$.

Cushing K, Alvarado DM, Ciorba MA. 2015. Butyrate and mucosal inflammation: new scientific evidence supports clinical observation. Clin Transl Gastroenterol. 6:e108.

Gidenne T, Carabaño R, García J, De Blas C. 2010. Fibre digestion. In: de Blas C, Wiseman J, editors. The nutrition of the rabbit. 2nd ed. Wallingford (UK): CABI Publishing CAB International; p. 66-82.

Goering HK, Van Soest PJ. 1970. Forage fibre analyses (apparatus, reagents, procedures and some applications). Agriculture handbook no. 379. Washington (DC): Agricultural Research Service, USDA.

Gómez-Conde MS, Rozas AP, de Badiola I, Pérez-Alba L, Blas C, de Carabaño R, García J. 2009. Effect of neutral detergent soluble fibre on digestion, intestinal microbiota and performance in twenty five day old weaned rabbits. Lives Sci. 125:192-198.

Guilloteau P, Martin L, Eeckhaut V, Ducatelle R, Zabielski R, van Immerseel F. 2010. From the gut to the peripheral tissues: the multiple effects of butyrate. Nutr Res Rev. 23:366-384.

Jiao LF, Song ZH, Ke YL, Xiao K, Hu CH, Shi B. 2014. Cello-oligosaccharide influences intestinal microflora, mucosal architecture and nutrient transport in weaned pigs. Anim Feed Sci Technol. 195:85-91.

Martínez-Vallespín B, Martínez-Paredes E, Ródenas L, Cervera C, Pascual JJ, Blas E. 2011. Combined feeding of rabbit female and young: partial replacement of starch with acid detergent fibre or/and neutral detergent soluble fibre at two protein levels. Lives Sci. 141:155-165.

Mateos I, Ranilla JM, Tejido ML, Saro C, Kamel C, Carro MD. 2013. The influence of diet on the effectiveness of garlic oil and cinnamaldehyde to manipulate in vitro ruminal fermentation and methane production. Anim Prod Sci. 53:299-307.

Mateos I, Ranilla MJ, Saro C, Carro MD. 2015. Comparison of fermentation characteristics and bacterial diversity in the rumen of sheep and batch cultures of rumen microorganisms. J Agric Sci. 153:1097-1106.

Mertens DR. 2002. Gravimetric determination of amylase-treated neutral detergent fiber in feeds with refluxing in beakers or crucibles: collaborative study. J AOAC Int. 85:1217-1240.

Ocasio-Vega C. 2018. Effect of cellobiose and dietary soluble fibre on fermentation, growth performance and health in rabbits [PhD thesis]. Spain: Universidad Politéctica de Madrid.

Ocasio-Vega C, Abad-Guamán R, Delgado R, Carabaño R, Carro MD, García J. 2018a. In vitro caecal fermentation of carbohydrate-rich feedstuffs in rabbits as affected by substrate predigestion and donors' diet. World Rabbit Sci. 26:15-25. doi:10.4995/wrs.2018.7854 
Ocasio-Vega C, Delgado R, Abad-Guamán R, Carabaño R, Carro MD, Menoyo D, García J. 2018b. The effect of cellobiose on the health status of growing rabbits depends on the dietary level of soluble fiber. J Anim Sci. doi:10.1093/jas/sky106

Olano-Martin E, Mountzouris KC, Gibson GR, Rastall RA. 2000. In vitro fermentability of dextran, oligodextran and maltodextrin by human gut bacteria. Br J Nutr. 83:247-255.

Pedersen MB, Yu S, Arent S, Dalsgaard S, Bach Knudsen KE, Lærke HN. 2015. Xylanase increased the ileal digestibility of nonstarch polysaccharides and concentration of low molecular weight nondigestible carbohydrates in pigs fed high levels of wheat distillers dried grains with solubles. J Anim Sci. 93:2885-2893.

Ramos MA, Carabaño R, Boisen S. 1992. An in vitro method for estimating digestibility in rabbits. J Appl Rabbit Res. 15:938.

Raqib R, Sarker P, Bergman P, Ara G, Lindh M, Sack DA, Nasirul Islam KM, Gudmundsson GH, Andersson J, Agerberth B. 2006. Improved outcome in shigellosis associated with butyrate induction of an endogenous peptide antibiotic. Proc Natl Acad Sci. 103:9178-9183. Epub 2006/06/03.

Rodríguez-Romero N, Abecia L, Fondevila M. 2012. Bacterial profile from caecal contents and soft faeces in growing rabbits given diets differing in soluble and insoluble fibre levels. Anaerobe. 18:602-607.

Rodríguez-Romero N, Abecia L, Fondevila M, Balcells J. 2011. Effects of levels of insoluble and soluble fibre in diets for growing rabbits on faecal digestibility, nitrogen recycling and in vitro fermentation. World Rabbit Sci. 19:85-94.

Schofield P, Pitt RE, Pell AN. 1994. Kinetics of fiber digestion from in vitro gas production. J Anim Sci. 72:2980-2991.

Song J, Jiao LF, Xiao K, Luan ZS, Hu CH, Shi B, Zhan XA. 2013. Cello-oligosaccharide ameliorates heat stress-induced impairment of intestinal microflora, morphology and barrier integrity in broilers. Anim Feed Sci Technol. 185:175-181.

Trocino A, García J, Carabaño R, Xiccato G. 2013. A meta-analysis on the role of soluble fibre in diets for growing rabbits. World Rabbit Sci. 21:1-15.

Van Zanten GC, Knudsen A, Röytiö H, Forssten S, Lawther M, Blennow A, Lahtinen SJ, Jakobsen M, Svensson B, Jespersen L. 2012. The effect of selected synbiotics on microbial composition and short-chain fatty acid production in a model system of the human colon. PLoS One. 7:e47212.

Vernay M. 1987. Origin and utilization of volatile fatty acids and lactate in the rabbit: influence of the faecal excretion pattern. Br J Nutr. 57:371-381.

Von Engelhardt W, Rönnau K, Rechkemmer G, Sakata T. 1989. Absorption of short-chain fatty acids and their role in the hindgut of monogastric animals. Anim Feed Sci Technol. 23:43-53.

Yang HJ, Cao YC, Zhang DF. 2010. Caecal fermentation patterns in vitro of glucose, cellobiose, microcrystalline cellulose and NDF separated from alfalfa hay in the adult rabbit. Anim Feed Sci Technol. 162:149-154. 